\title{
Body Image of Patients with Breast Cancer after Breast Conserving Surgery and Mastectomy: A Comparative Study
}

\author{
Asiie Olfatbakhsh ${ }^{1,}{ }^{*}$, Esmatosadat Hashemi ${ }^{1}$, Ahmad Kaviani ${ }^{2}$, \\ Fateme Sari ${ }^{1}$, HodaTafazzoli-Harandi ${ }^{1}$
}

1 Breast Disease Department, Breast Cancer Research Center, Motamed Cancer Institute, ACECR, Tehran, Iran

2 Department of Surgery, Tehran University of Medical Sciences, Tehran, Iran

* Corresponding author: Asiie Olfatbakhsh, Breast Disease Department, Breast

DOI: $10.21859 /$ mci-supp-103 Cancer Research Center, Motamed Cancer Institute, ACECR, Tehran, Iran. E-mail: folfatbakhsh@yahoo.com

\section{Keywords:}

Breast Cancer

Breast Conserving Surgery

Mastectomy

\begin{abstract}
Introduction: Altered appearance results in substantial distress in breast cancer patients. This study compares the effects of BCS or MRMon BI of patients in Breast Cancer Research Center (BCRC) and the associated factors.

Materials and Methods: The subjects were selected of patients at least 6 months after starting follow up. We used Iranian version of the BICI questionnaire. They asked about their satisfaction of the surgery results (score 0-10), socioeconomic and clinical characteristics. Statistical evaluations were made using chi-square test and independent samples t-test. Data were analyzed using SPSS ${ }^{\circledR}$. All tests were considered significant at $\mathrm{P}$ values $=$ 0.05 .

Results: Overall 140 patients were included $(\mathrm{BCS}=70$ and $\mathrm{MRM}=70)$. The mean age of patients was 44.96 y (24-62). In all, $85.7 \%$ were married, $76.3 \%$ housewife, in $52.1 \%$ the family income was between $10-30$ million RLs, $60.9 \%$ were living in big cities, and in $72.9 \%$ the education level was diploma or lower. There were no significant difference between two groups about age and other characteristics except for marital status $(P=0.03)$. The BI scale was significantly different between BCS and MRM groups (33.8 vs. 39.53, $\mathrm{P}=0.007)$. After division the BI scale to Appearance dissatisfied and Interference with social activity, again there were significant difference between two groups $(\mathrm{P}=0.19$ and $\mathrm{P}$ $=0.007$ respectively). The mean satisfaction score of the surgery result were 9.49 for BCS and 8.97 for MRM groups $(\mathrm{P}=0.03)$. None of the characteristic factors like age, marital status, education, economic status, job or place of residence had impact on the BI scale. Conclusions: The results of this studyshowed that body imageperception is significantly better in patients with breast cancer undergoing BCS and that was unrelated to age, socioeconomic or marital status of them.
\end{abstract}

